\title{
Closed-Orbit Theory of Spatial Density Oscillations in Finite Fermion Systems
}

\author{
Jérôme Roccia and Matthias Brack
}

Institut für Theoretische Physik, Universität Regensburg, D-93040 Regensburg, Germany

(Received 4 March 2008; revised manuscript received 22 April 2008; published 23 May 2008)

\begin{abstract}
We investigate the particle and kinetic-energy densities for $N$ noninteracting fermions confined in a local potential. Using Gutzwiller's semiclassical Green function, we describe the oscillating parts of the densities in terms of closed nonperiodic classical orbits. We derive universal relations between the oscillating parts of the densities for potentials with spherical symmetry in arbitrary dimensions and a "local virial theorem" valid also for arbitrary nonintegrable potentials. We give simple analytical formulas for the density oscillations in a one-dimensional potential.
\end{abstract}

DOI: 10.1103/PhysRevLett.100.200408

Introduction. - Finite systems of fermions are studied in many branches of physics, e.g., electrons in atoms, molecules, and quantum dots, protons and neutrons in atomic nuclei, or fermionic atoms in traps. Common to these systems are pronounced shell effects which result from the combination of quantized energy spectra with the Pauli exclusion principle. The shell effects manifest themselves most clearly in ionization (or separation) energies and total binding energies. They lead to "magic numbers" of particles in particularly stable species, when degenerate shells or approximately degenerate bunches of singleparticle levels are filled. Shell effects appear also in spatial particle densities [1,2] and kinetic-energy densities. Near the center of a system, the alternating parities of the occupied shells lead to regular quantum oscillations, while the so-called "Friedel oscillations" characteristically appear near the surface of a sufficiently steep confining potential. Kohn and Sham [1] analyzed both oscillations in the particle density using Green functions in the onedimensional WKB approximation. Thorpe and Thouless [2] extended their method to the central oscillations in three-dimensional systems with radial symmetry.

In the periodic orbit theory (POT) [3-5], semiclassical "trace formulas" allow one to relate the level density of a quantized Hamiltonian system to the periodic orbits of the corresponding classical system. This can be used to interpret quantum shell effects occurring in finite fermion systems in terms of the shortest periodic orbits (see Ref. [6] for an introduction to POT and applications to various branches of physics). To our knowledge, no attempt has been made thus far to interpret quantum oscillations of spatial densities in terms of classical orbits. In this Letter, we use the semiclassical Green function of Gutzwiller [3] to derive analytical expressions for the oscillating parts of particle and kinetic-energy densities in terms of closed nonperiodic classical orbits.

General framework. - We consider a $D$-dimensional system of $N$ noninteracting particles with mass $m$, which obey Fermi-Dirac statistics and are bound by a local potential $V(\mathbf{r})$. Note that $V(\mathbf{r})$ can be the self-consistent mean
PACS numbers: 05.30.Fk, 03.65.Sq, 03.75.Ss, 71.10.-w

field of an interacting fermion system (such as a nucleus). The energy eigenvalues $E_{n}$ and eigenfunctions $\psi_{n}(\mathbf{r})$ are given by the stationary Schrödinger equation. The particle density of the system at zero temperature, ignoring the spin degeneracy, is given by

$$
\rho(\mathbf{r})=\sum_{E_{n} \leq \lambda(N)} \psi_{n}^{\star}(\mathbf{r}) \psi_{n}(\mathbf{r}),
$$

where the Fermi energy $\lambda(N)$ is determined by normalizing the density to the given particle number $N$. For the kineticenergy density we discuss two different forms,

$$
\begin{gathered}
\tau(\mathbf{r})=-\frac{\hbar^{2}}{2 m} \sum_{E_{n} \leq \lambda} \psi_{n}^{\star}(\mathbf{r}) \nabla^{2} \psi_{n}(\mathbf{r}), \\
\tau_{1}(\mathbf{r})=\frac{\hbar^{2}}{2 m} \sum_{E_{n} \leq \lambda}\left|\nabla \psi_{n}(\mathbf{r})\right|^{2},
\end{gathered}
$$

which after integration both lead to the exact total kinetic energy. We rewrite the above densities in the form

$$
\begin{gathered}
\rho(\mathbf{r})=-\left.\frac{1}{\pi} \operatorname{Im} \int_{0}^{\lambda} d E G\left(E, \mathbf{r}, \mathbf{r}^{\prime}\right)\right|_{\mathbf{r}^{\prime}=\mathbf{r}}, \\
\tau(\mathbf{r})=\left.\frac{\hbar^{2}}{2 \pi m} \operatorname{Im} \int_{0}^{\lambda} d E \nabla_{\mathbf{r}^{\prime}}^{2} G\left(E, \mathbf{r}, \mathbf{r}^{\prime}\right)\right|_{\mathbf{r}^{\prime}=\mathbf{r}}, \\
\tau_{1}(\mathbf{r})=-\left.\frac{\hbar^{2}}{2 \pi m} \operatorname{Im} \int_{0}^{\lambda} d E \nabla_{\mathbf{r}} \cdot \nabla_{\mathbf{r}^{\prime}} G\left(E, \mathbf{r}, \mathbf{r}^{\prime}\right)\right|_{\mathbf{r}^{\prime}=\mathbf{r}},
\end{gathered}
$$

where $G\left(E, \mathbf{r}, \mathbf{r}^{\prime}\right)$ is the Green function in the energy representation

$$
G\left(E, \mathbf{r}, \mathbf{r}^{\prime}\right)=\sum_{n} \frac{\psi_{n}^{\star}(\mathbf{r}) \psi_{n}\left(\mathbf{r}^{\prime}\right)}{E+i \epsilon-E_{n}} \quad(\epsilon>0),
$$

and the identity $1 /\left(E+i \epsilon-E_{n}\right)=\mathcal{P}\left[1 /\left(E-E_{n}\right)\right]-$ $i \pi \delta\left(E-E_{n}\right)$ is used ( $\mathcal{P}$ is the Cauchy principal value).

To obtain semiclassical expressions, we replace the Green function by Gutzwiller's approximation [3] 


$$
G_{\text {scl }}\left(E, \mathbf{r}, \mathbf{r}^{\prime}\right)=\alpha_{D} \sum_{\text {cl. trj. }} \sqrt{|\bar{D}|} e^{(i / h) S\left(E, \mathbf{r}, \mathbf{r}^{\prime}\right)-i \mu(\pi / 2)},
$$

which is valid to leading order in $1 / \hbar$ in the semiclassical limit $\hbar \rightarrow 0$, i.e., when the dominating classical actions $S\left(E, \mathbf{r}, \mathbf{r}^{\prime}\right)$ are large compared with $\hbar$. In Eq. (8), $\mathcal{D}$ is the Van Vleck determinant given below, $\mu$ is the Morse index, and $\alpha_{D}=2 \pi(2 i \pi h)^{-(D+1) / 2}$. The sum is over all classical trajectories starting at $\mathbf{r}$ and ending at $\mathbf{r}^{\prime}$. The action integral along each trajectory is

$$
S\left(E, \mathbf{r}, \mathbf{r}^{\prime}\right)=\int_{\mathbf{r}}^{\mathbf{r}^{\prime}} \mathbf{p}\left(\mathbf{r}^{\prime \prime}\right) \cdot d \mathbf{r}^{\prime \prime}
$$

Since we have to use $\mathbf{r}=\mathbf{r}^{\prime}$ in (4)-(6), only closed trajectories starting and ending at the same point $\mathbf{r}$ have to be included in the sum of (8). Following Gutzwiller [3], we use for each trajectory a local coordinate system $\mathbf{r}=$ $\left(q, \mathbf{r}_{\perp}\right)=\left(q, r_{\perp 1}, r_{\perp 2}, \ldots, r_{\perp(D-1)}\right)$, whose first variable $q$ is chosen along the trajectory, while the vector $\mathbf{r}_{\perp}$ of the remaining $D-1$ variables is transverse to it. The Van Vleck determinant then becomes

$$
\mathcal{D}=\frac{(-1)^{D} m^{2} \mathcal{D}_{\perp}}{p(E, \mathbf{r}) p\left(E, \mathbf{r}^{\prime}\right)}, \quad \mathcal{D}_{\perp}=\operatorname{det}\left(\partial \mathbf{p}_{\perp}^{\prime} / \partial \mathbf{r}_{\perp}\right)
$$

where $\mathbf{p}(E, \mathbf{r})=\dot{\mathbf{r}}|\sqrt{2 m[E-V(\mathbf{r})]}| /|\dot{\mathbf{r}}|$ is the classical momentum and $p(E, \mathbf{r})=m \dot{q}(E, \mathbf{r})$ its modulus.

We now want to keep only the leading-order terms in the semiclassical expansion parameter $\hbar$. To this purpose it is useful to decompose the Fermi energy into a smooth and an oscillating part: $\lambda=\tilde{\lambda}+\delta \lambda$. Assuming that $\delta \lambda \ll \tilde{\lambda}$, one can show [7] that

$$
\delta \lambda=-\int_{0}^{\tilde{\lambda}} \delta g(E) d E / \tilde{g}(\tilde{\lambda}), \quad \int_{0}^{\tilde{\lambda}} \tilde{g}(E) d E=N,
$$

where $\tilde{g}(E)$ is the smooth part of the level density and $\delta g(E)$ its oscillating part, semiclassically given by a sum over the periodic orbits of the classical system [3].

The sum over closed trajectories to be used in (4)-(6) can be separated into a sum over periodic orbits (POs) and a sum over nonperiodic orbits (NPOs). The actions along the POs are independent of $\mathbf{r}$; their contributions are therefore smooth functions, given only by the initial and final momenta $\mathbf{p}=\mathbf{p}(E, \mathbf{r})$ and $\mathbf{p}^{\prime}=\mathbf{p}\left(E, \mathbf{r}^{\prime}\right)$. To lowest order in $\hbar$, the semiclassical densities are given by the POs with zero length. They are identical with the smooth ThomasFermi (TF) densities [8], like it is known [9] for the level density $\tilde{g}(E)=g_{\mathrm{TF}}(E)$. To next order in $\hbar$, the sums over all NPOs yield the density oscillations, so that the semiclassical particle density has the form

$$
\rho_{\mathrm{scl}}(\mathbf{r})=\rho_{\mathrm{TF}}(\mathbf{r})+\delta \rho(\mathbf{r}) \text {. }
$$

Analogous forms hold for $\tau(\mathbf{r})$ and $\tau_{1}(\mathbf{r})$

For the kinetic-energy densities we have to derive the Green function (8) twice according to (5) and (6). The semiclassically leading terms come from the derivatives of $S\left(E, \mathbf{r}, \mathbf{r}^{\prime}\right)$, for which the relations $\nabla_{\mathbf{r}^{\prime}} S\left(E, \mathbf{r}, \mathbf{r}^{\prime}\right)=\mathbf{p}^{\prime}$ and $\nabla_{\mathbf{r}} S\left(E, \mathbf{r}, \mathbf{r}^{\prime}\right)=-\mathbf{p}$ hold. The energy integration in Eqs. (4)-(6) can be done by parts. The leading-order results come from the upper integration limit, taken as $\tilde{\lambda}$. The lower limit, which must be taken to be $V(\mathbf{r})$ since in the semiclassical approximation one has to stay in the classically allowed region, gives no contributions. We then obtain for the oscillating parts of the densities

$$
\begin{gathered}
\delta \rho(\mathbf{r})=\frac{m \hbar}{\pi} \operatorname{Re} \alpha_{D} \sum_{\mathrm{NPO}} \frac{\sqrt{\left|\overline{\mathcal{D}} \bar{\perp}_{1}\right|_{\mathbf{r}^{\prime}=\mathbf{r}}}}{p(\tilde{\lambda}, \mathbf{r}) T(\tilde{\lambda}, \mathbf{r})} e^{i \Phi(\tilde{\lambda}, \mathbf{r})} \\
\delta \tau(\mathbf{r})=\frac{\hbar}{2 \pi} \operatorname{Re} \alpha_{D} \sum_{\mathrm{NPO}} \frac{p(\tilde{\lambda}, \mathbf{r}) \sqrt{\left|\mathcal{D}_{\perp}\right|_{\mathbf{r}^{\prime}=\mathbf{r}}}}{T(\tilde{\lambda}, \mathbf{r})} e^{i \Phi(\tilde{\lambda}, \mathbf{r})}, \\
\delta \tau_{1}(\mathbf{r})=\frac{\hbar}{2 \pi} \operatorname{Re} \alpha_{D} \sum_{\mathrm{NPO}} \frac{\left\{\left(\mathbf{p} \cdot \mathbf{p}^{\prime}\right)_{\lambda} \sqrt{\left|\overline{\mathcal{D}} \bar{D}_{\perp}\right|}\right\}_{\mathbf{r}^{\prime}=\mathbf{r}}}{p(\tilde{\lambda}, \mathbf{r}) T(\tilde{\lambda}, \mathbf{r})} e^{i \Phi(\tilde{\lambda}, \mathbf{r})}
\end{gathered}
$$

where $\left(\mathbf{p} \cdot \mathbf{p}^{\prime}\right)_{\lambda}=\mathbf{p}(\tilde{\lambda}, \mathbf{r}) \cdot \mathbf{p}\left(\tilde{\lambda}, \mathbf{r}^{\prime}\right)$, the phase function in the exponents is $\Phi(\tilde{\lambda}, \mathbf{r})=S(\tilde{\lambda}, \mathbf{r}, \mathbf{r}) / \hbar-\mu \frac{\pi}{2}$, and $T(\tilde{\lambda}, \mathbf{r})=d S(E, \mathbf{r}, \mathbf{r}) /\left.d E\right|_{E=\tilde{\lambda}}$. Since the modulus $p$ depends only on position and Fermi energy, but not on the orbits, we can take it outside the sum over the NPOs. We thus immediately find the general relation

$$
\delta \tau(\mathbf{r})=[\tilde{\lambda}-V(\mathbf{r})] \delta \rho(\mathbf{r}) .
$$

It holds for arbitrary, integrable or nonintegrable, local potentials in arbitrary dimensions. Equation (16) may be termed a "local virial theorem" because it relates kinetic and potential energy densities locally at any point. For $\delta \tau_{1}(\mathbf{r})$ we have no such relation, since it depends on the relative directions of final and initial momentum of each orbit. Because of the semiclassical nature of our approximation, Eq. (16) and the results derived below are expected to be valid in the limit of large particle numbers $N$.

One-dimensional systems. - For further development we now focus on one-dimensional systems characterized by a smooth binding potential $V(x)$ with a minimum at $x=0$. We will explicitly derive a semiclassical expression for the particle density $\rho(x)$; analogous results for the kinetic densities are found in the same way.

The classical motion at fixed energy $E$ is limited by the turning points $x_{ \pm}(E)$ defined by $V\left(x_{ \pm}\right)=E$, with $x_{+}(E)>$ 0 and $x_{-}(E)<0$. In one dimension there are only two types of trajectories going from $x$ to $x^{\prime}$ : the first type has its momenta at the initial and final points in the same direction, while for the second type they go in opposite directions. Without loss of generality we may choose $x_{-} \leq$ $x \leq x^{\prime} \leq x_{+}$. The shortest trajectory of the first type goes from $x$ directly to $x^{\prime}$ without reaching any of the turning points; it is indexed by the subscript " 0 " and has the action

$$
S_{0}\left(E, x, x^{\prime}\right)=S\left(E, 0, x^{\prime}\right)-S(E, 0, x) .
$$

All other trajectories of the first type bounce $j=1,2, \ldots$ times forth and back between the turning points before 
reaching $x^{\prime}$; they are indexed by " 1 " and have the actions

$$
S_{1 \pm}\left(E, x, x^{\prime}\right)=j S_{1}(E) \pm S_{0}\left(E, x, x^{\prime}\right) \quad(j=1,2, \ldots),
$$

where $S_{1}(E)$ is the action of the primitive periodic orbit and the sign \pm refers to the starting direction. The trajectories of the second type bounce $k=0,1, \ldots$ times forth and back before reaching $x^{\prime}$; they are indexed by " 2 " and have the actions

$$
\begin{aligned}
S_{2 \pm}\left(E, x, x^{\prime}\right)= & k S_{1}(E)+2 S_{\bar{\mp}}(E) \pm\left[S\left(E, 0, x^{\prime}\right)\right. \\
& +S(E, 0, x)] \quad(k=0,1, \ldots),
\end{aligned}
$$

where $S_{-}(E)=S\left(E, x_{-}, 0\right)$ and $S_{+}(E)=S\left(E, 0, x_{+}\right)$. For a symmetric potential with $V(x)=V(-x)$, one has $S_{-}(E)=$ $S_{+}(E)=S_{1}(E) / 2$. From (10) we have $\sqrt{|\mathcal{D}(E, x, x)|}=$ $m / p(E, x)$ for all trajectories. For smooth potentials in one dimension, the Morse index $\mu$ is equal to the number of turning points, which for the above trajectories is $\mu_{0}=$ $0, \mu_{1 \pm}=2 j$, and $\mu_{2 \pm}=2 k+1$. (For a one-dimensional box with reflecting walls, the Morse index equals twice the number of turning points; our semiclassical densities become exact in this case.)

Using (17)-(19) and $D=1$ in Eq. (8), we now obtain the semiclassical particle density $\rho_{\text {scl }}(x)$ as a sum of the three types of contributions indexed as above:

$$
\rho_{\mathrm{scl}}(x)=\rho_{0}(x)+\sum_{\sigma=+-}\left[\rho_{1 \sigma}(x)+\rho_{2 \sigma}(x)\right] .
$$

Since we have to use $x=x^{\prime}$, the only contributing orbits of type 0 have zero length, those of type 1 are periodic, and those of type 2 are nonperiodic. Doing the energy integration by parts, we get to leading-order in $\hbar$

$$
\begin{gathered}
\rho_{0}(\lambda, x)=\frac{(2 m)^{1 / 2}}{\pi \hbar} \sqrt{\lambda-V(x)}, \\
\rho_{1}(x)=\frac{2 m}{\pi} \sum_{j=1}^{\infty}(-1)^{j} \frac{\sin \left\{j S_{1}(\tilde{\lambda}) / \hbar\right\}}{p(\tilde{\lambda}, x) j T_{1}(\tilde{\lambda})},
\end{gathered}
$$

where $T_{1}(\tilde{\lambda})$ is the period of the primitive periodic orbit. Taylor expanding $\rho_{0}(\lambda, x)$ in Eq. (21) around $\tilde{\lambda}$ yields the well-known TF density, $\rho_{\mathrm{TF}}(x)=\rho_{0}(\tilde{\lambda}, x)$, plus a term linear in $\delta \lambda$ which, using Eq. (11), cancels exactly the contribution $\rho_{1}(x)$ in (22). The leading-order oscillating term is therefore given by the type 2 orbits, i.e., by $\delta \rho(x)=$ $\rho_{2+}(x)+\rho_{2-}(x)$ which has the explicit form

$$
\delta \rho(x)=-\frac{m}{\pi} \sum_{\substack{k=0 \\ \sigma= \pm \pm}}^{\infty}(-1)^{k} \frac{\cos \left\{\left[k S_{1}(\tilde{\lambda})+R_{\sigma}(\tilde{\lambda}, x)\right] / \hbar\right\}}{p(\tilde{\lambda}, x)\left[k T_{1}(\tilde{\lambda})+R_{\sigma}^{\prime}(\tilde{\lambda}, x)\right]},
$$

with $R_{ \pm}(\tilde{\lambda}, x)=2 S_{ \pm}(\tilde{\lambda}) \mp 2 S(\tilde{\lambda}, 0, x)$. This result is equivalent, although not obviously identical, with the result given in Eq. (3.36) of [1].
For the kinetic-energy densities we proceed in the same way. The smooth parts of $\tau(x)$ and $\tau_{1}(x)$ are identical and equal to the TF kinetic-energy density $\tau_{\mathrm{TF}}(x)$; for their oscillating parts we obtain the one-dimensional version of the relation (16) and, in addition, the new relation

$$
\delta \tau_{1}(x)=-\delta \tau(x),
$$

which holds due to the opposite initial and final momenta of the NPOs of type 2 which contribute to (15).

In Fig. 1, we test our semiclassical results for the potential $V(x)=x^{4} / 4$ with $N=40$ particles (with units such that $\hbar=m=1)$. The upper panel shows $\delta \rho(x)$ given in (23) by the solid line, while the dots represent the quantummechanical expression (1) after subtracting the TF density. The agreement is very good except close to the classical turning point where the TF approximation breaks down. The lower panel demonstrates the validity of the relations (16) (with $\mathbf{r} \rightarrow x$ ) and (24). The small deficiencies near the classical turning points can be overcome and the tail in the classically forbidden region described by the standard WKB treatment [1,2] or the TF-Weizsäcker theory [8].

A simpler form for $\delta \rho(x)$ is found if one restricts oneself to the interior part of the system around $x=0$, where $V(x) \ll \tilde{\lambda}$. Then the action integral $S(\tilde{\lambda}, 0, x)$ can be approximated by $S(\tilde{\lambda}, 0, x) \simeq x p_{\lambda}$, where $p_{\lambda}=(2 m \tilde{\lambda})^{1 / 2}$ is

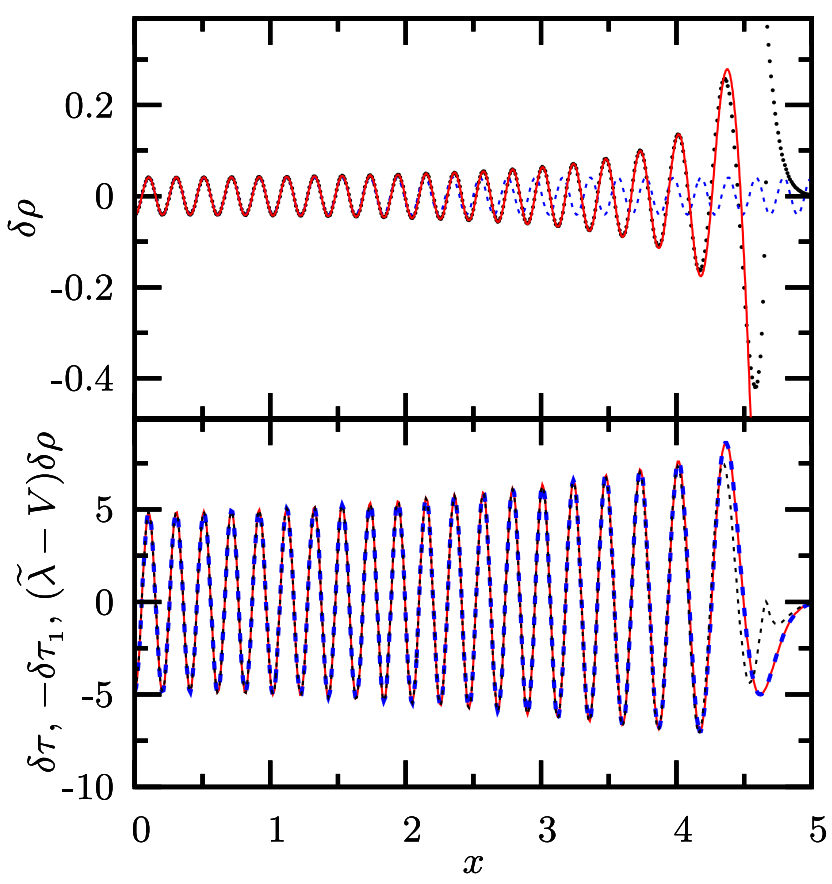

FIG. 1 (color online). Upper panel: Oscillating part $\delta \rho(x)$ of the particle density of $N=40$ fermions in the potential $V(x)=$ $x^{4} / 4$. Dots show the quantum-mechanical result; the solid line shows the semiclassical result $\delta \rho(x)$ in (23) and the dashed line the approximation (25) for small $x$ values. Lower panel: Oscillating parts of the quantum-mechanical kinetic-energy densities in the same system: $\delta \tau(x)$ (solid line) and $-\delta \tau_{1}(x)$ (dashed line). The dotted line shows the function $[\tilde{\lambda}-V(x)] \delta \rho(x)$ using the quantum-mechanical $\delta \rho(x)$. 
the smooth Fermi momentum. Using the fact that the action $S_{1}(\tilde{\lambda})$ in one dimension can be related to the particle number $N$ by $S_{1}(\tilde{\lambda}) \approx 2 \pi \hbar N$, which is nothing but the well-known Bohr-Sommerfeld quantization rule, and using the identity $\sum_{k=0}^{\infty}(-1)^{k} \cos [(2 k+1) N] /(2 k+1)=$ $(-1)^{N} \pi / 4$, we find the approximate expression for the central oscillations:

$$
\delta \rho(x)=(-1)^{N+1} \frac{m}{p_{\lambda} T_{1}(\tilde{\lambda})} \cos \left(2 x p_{\lambda} / \hbar+\delta \Phi\right),
$$

where $\delta \Phi=\left[S_{-}(\tilde{\lambda})-S_{+}(\tilde{\lambda})\right] / \hbar$ is a phase difference related to the asymmetry of the potential. Equation (25) can also be obtained from Eq. (3.36) in Ref. [1] in the limit $V(x) \ll \tilde{\lambda}$. It is shown by the dashed line in the upper panel of Fig. 1. Using Eqs. (16) and (24) one can give analogous simple results for the kinetic-energy density oscillations near $x=0$.

Our derivation shows that periodic orbits do not contribute to the oscillations in the densities $\rho(x), \tau(x)$, and $\tau_{1}(x)$, while they are known [3] to give the most important contributions to the oscillating level density $\delta g(E)$. In fact, the most important contribution to (23) comes from the two shortest nonperiodic orbits which go from $x$ to one of the turning points and back; for small $x$ their action difference is $2 x p_{\lambda}$. The summation over all longer nonperiodic orbits yields the oscillating sign depending on the particle number $N$. We emphasize that the oscillations in Eq. (25) have the universal wavelength $\hbar \pi / p_{\lambda}$ independent of the particular form of the potential $V(x)$.

Higher-dimensional radial systems. - In a forthcoming paper [10], we generalize our method for higherdimensional systems. For binding potentials $V(r)$ with spherical symmetry in $D>1$ dimensions, one can separate two kinds of spatial oscillations in the radial variable: (i) irregular longer-ranged oscillations, which are attributed to nonlinear classical orbits (and are absent in spherical harmonic oscillators) and (ii) regular, rapid oscillations of the kind discussed above and denoted here by $\delta \rho(r)$, $\delta \tau(r)$, and $\delta \tau_{1}(r)$. The regular rapid oscillations originate from nonperiodic linear orbits with zero angular momentum, starting from $r$ in the radial direction and returning with opposite radial momentum to $r$; these orbits correspond exactly to the above type 2 orbits in one dimension. From their contributions to Eq. (8) and hence to Eq. (13), it is straightforward to derive the following relation, valid to leading order in $\hbar$ :

$$
-\frac{\hbar^{2}}{8 m} \nabla^{2} \delta \rho(r)=[\tilde{\lambda}-V(r)] \delta \rho(r) .
$$

Similarly, it follows from the nature of the radial type 2 orbits that $\left(\mathbf{p} \cdot \mathbf{p}^{\prime}\right)_{\mathbf{r}^{\prime}=\mathbf{r}}=-p^{2}$ in (15) and hence the rapid oscillations in the kinetic-energy densities $\tau(r)$ and $\tau_{1}(r)$ fulfill the relation (24) in the radial variable $r$ :

$$
\delta \tau_{1}(r)=-\delta \tau(r)
$$

For small $r$, where $V(r) \ll \tilde{\lambda}$, Eq. (26) becomes a universal eigenvalue equation for $\delta \rho(r)$ with eigenvalue $\tilde{\lambda}$, which can be transformed into the Bessel equation. Its solutions yield the generalization of Eq. (25) (with $\delta \Phi=0$ ) for the rapid oscillations near $r=0$ :

$$
\delta \rho(r)=(-1)^{M-1} \frac{m}{2 \hbar T_{r 1}(\tilde{\lambda})}\left(\frac{p_{\lambda}}{4 \pi \hbar r}\right)^{\nu} J_{\nu}\left(2 r p_{\lambda} / \hbar\right) .
$$

Here $J_{\nu}(z)$ is a Bessel function with index $\nu=D / 2-1$, $M$ is the number of filled main shells, and $T_{r 1}$ is the period of one radial oscillation. For $D=3$, Eq. (28) agrees with the result of [2] up to a $\tilde{\lambda}$ dependent normalization factor. Our results (16) and (26)-(28) agree with those derived analytically for harmonic oscillator potentials $V(r)=c r^{2}$ in arbitrary dimension $D$ from the quantum-mechanical densities to leading order in a $1 / N$ expansion [11]. Numerical tests of our semiclassical relations for a variety of systems will be given in Ref. [10].

Conclusions. - We have shown that quantum oscillations in spatial densities can be derived without resorting to wave functions, but using the closed nonperiodic orbits of the classical system. Our one-dimensional result for $\delta \rho(x)$ is equivalent to that of [1], but its derivation by the summation over classical orbits appears more transparent to us. Our results may become useful in the analysis of weakly interacting trapped fermionic gases (see, e.g., [12]) for which the mean-field approximation is appropriate. We present it as a challenge to verify the "local virial theorem" (16) experimentally.

We are grateful to J. D. Urbina for helpful comments and to A. Koch for numerical data used in the figure.

[1] W. Kohn and L. J. Sham, Phys. Rev. 137, A1697 (1965).

[2] M. A. Thorpe and D. J. Thouless, Nucl. Phys. A156, 225 (1970).

[3] M. C. Gutzwiller, J. Math. Phys. (N.Y.) 12, 343 (1971); M.C. Gutzwiller, Chaos in Classical and Quantum Mechanics (Springer-Verlag, New York, 1990).

[4] R. Balian and C. Bloch, Ann. Phys. (N.Y.) 69, 76 (1972).

[5] Special Issue on Periodic Orbit Theory, edited by $\mathrm{P}$. Cvitanović [Chaos 2, 1 (1992)].

[6] M. Brack and R. K. Bhaduri, Semiclassical Physics (Westview, Boulder, CO, 2003).

[7] M. Centelles, P. Leboeuf, A. Monastra, J. Roccia, P. Schuck, and X. Viñas, Phys. Rev. C 74, 034332 (2006).

[8] N. March, Adv. Phys. 6, 1 (1957).

[9] M. Berry and K.E. Mount, Rep. Prog. Phys. 35, 315 (1972).

[10] J. Roccia, A. Koch, M. Brack, and M. V. N. Murthy (to be published).

[11] M. Brack and M. V. N. Murthy, J. Phys. A36, 1111 (2003).

[12] B. DeMarco and D. S. Jin, Science 285, 1703 (1999). 